$G D$

Maximum Entropy Distribution and Closure for

Bose-Einstein and Fermi-Dirac Radiation Transport

J. Cernohorsky and S.A. Bludman

March 1994

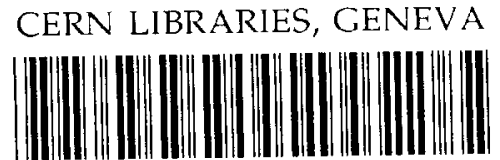

P00024173

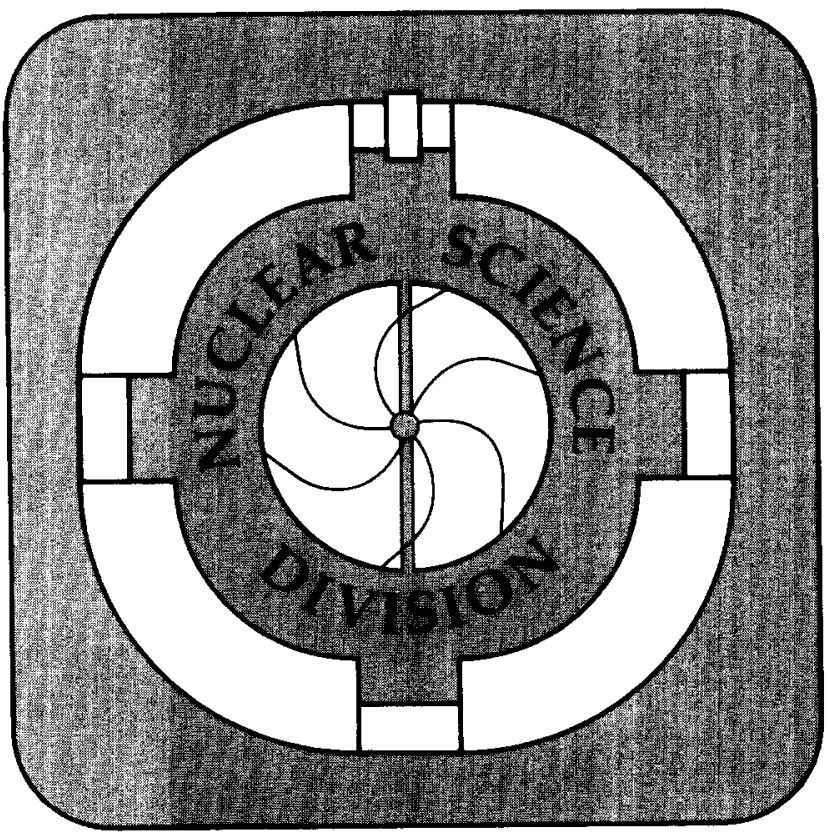

Prepared for the U.S. Department of Energy under Contract Number DE-AC03-76SF00098 
LBL - 34920Rev.

UPR - 971T

\title{
Maximum Entropy Distribution and Closure for Bose-Einstein and Fermi-Dirac Radiation Transport ${ }^{\dagger}$
}

\author{
J. Cernohorsky ${ }^{\ddagger}$ and S.A. Bludman ${ }^{\S}$ \\ Nuclear Science Division \\ Lawrence Berkeley Laboratory \\ University of California \\ Berkeley, California 94720, U.S.A.
}

March 17, 1994

\footnotetext{
${ }^{\dagger}$ This work was supported by the Director, Office of Energy Research, Office of High Energy and Nuclear Physics, Division of Nuclear Physics, of the U.S. Department of Energy under Contract DE-AC03-76SF00098 and by the Department of Energy contract AC02-76-ERO-3071 at the University of Pennsylvania.

$\ddagger$ On leave from the Max-Planck Institut für Astrophysik, 85748 Garching, Federal Republic of Germany

${ }^{\S}$ Center for Particle Astrophysics, University of California, Berkeley, California 94720 and Department of Physics, University of Pennsylvania, Philadelphia, PA 19104 U.S.A.
} 


\title{
MAXIMUM ENTROPy Distribution AND Closure for Bose-Einstein And FERmi-Dirac Radiation Transport
}

\author{
J. Cernohorsky \\ Lawrence Berkeley Laboratory, University of California, Berkeley CA 94720, U.S.A. \\ On leave from the Max-Planck Institut für Astrophysik, 85748 Garching, Federal \\ Republic of Germany
}

S.A. Bludman

Center for Particle Astrophysics and Lawrence Berkeley Laboratory, Berkeley CA 94709, Department of Physics, University of Pennsylvania, Philadelphia, PA 19104

March 17, 1994

\begin{abstract}
We derive and study the variable Eddington factors following without approximation from the maximum entropy distribution. A unified formalism is developed for classical Maxwell-Boltzmann, Bose-Einstein, and Fermi-Dirac radiation. In the classical and high occupancy limits the maximum entropy closure is bounded by previously known variable Eddington factors that depend only on the flux: the Levermore-Pomraning closure for Bose radiation at high occupation density, and Minerbo's flux-limiter for the Boltzmann limit of either quantum statistics. For intermediate occupancy, the maximum entropy closure depends on both the occupation density and the flux. The Fermi-Dirac maximum entropy variable Eddington factor exhibits scale invariance, which leads to a simple, analytic closure for fermion radiation. This Fermi-Dirac variable Eddington factor agrees well with Monte Carlo neutrino transport closures during early stages of neutron star formation.
\end{abstract}




\section{Variable Eddington Factors to Close the Hierarchy of Angular Moment Equations}

The maximal information on any radiation field is contained in the distribution function $\mathcal{F}\left(x^{\mu}, p^{\mu}\right)$ which is the solution of the Boltzmann equation

$$
q^{\mu} \mathcal{F}_{; \mu}=C[\mathcal{F}]
$$

as function of space-time and four-momentum. The interaction between the radiation and the material medium through which it is propagating, including stimulated emission or absorption due to the Bose or Fermi statistics of the radiation, is described by the collision integral $C[\mathcal{F}]$, which is the only place through which the radiation quantum statistics enter the problem. For the most part, the geometry of the problem enters through the advective terms on the left hand side and through the boundary conditions.

Although there are problems for which it is feasible to solve the Boltzmann equation outright, this is invariably computationally very intensive. Moreover, the full distribution function $\mathcal{F}\left(x^{\mu}, p^{\mu}\right)$ often contains more information than is strictly required. In radiation hydrodynamics therefore, instead of the Boltzmann equation its angular moments are often considered. By integrating over the direction $\boldsymbol{\Omega}$ of the particle momentum, information on the angular dependence of the distribution is degraded, but the computational problem is significantly reduced.

Each angular moment equation of the Boltzmann equation contains angular moments of higher order. Any truncated hierarchy of moment equations contains more variables than equations and must be supplied with an additional equation, the closure. Usually, only the first two angular moments of the Boltzmann equation are considered, since these directly correspond to physical conservation laws of energy and momentum. They contain as variables the first and second Eddington factors,

$$
\begin{aligned}
\mathbf{f}\left(\omega, x^{\mu}\right) \equiv \mathbf{F} / e & =(1 / 4 \pi e) \int d \Omega \Omega \mathcal{F}\left(\omega, \Omega, x^{\mu}\right) \\
\overrightarrow{\mathbf{p}}\left(\omega, x^{\mu}\right) \equiv \overrightarrow{\mathbf{P}} / e & =(1 / 4 \pi e) \int d \Omega \Omega \Omega \mathcal{F}\left(\omega, \Omega, x^{\mu}\right)
\end{aligned}
$$

which are the flux and the pressure, normalised by the zeroeth Eddington factor

$$
e\left(\omega, x^{\mu}\right)=(1 / 4 \pi) \int d \Omega \mathcal{F}\left(\omega, \Omega, x^{\mu}\right),
$$


corresponding to the occupation density. If the variable Eddington factor, $\overrightarrow{\mathbf{p}}=\overrightarrow{\mathbf{p}}(\mathbf{f}, e)$ were known as function of the flux $f$ and occupation density $e$, the hierarchy of moment equations would be closed with the first two moments.

A practicable closure of the energy and momentum balance equations together with the variable Eddington factor should be easier to solve than the original Boltzmann equation and the results should approximate, as closely as possible, the angular moments of the exact distribution function $\mathcal{F}$, for each point in space-time $x^{\mu}$, and for every particle energy $\omega$. Beyond this 'weak equivalence' of the first three angular moments, a 'strong equivalence' of some model distribution to the exact distribution might be considered. If the closure is based on a model distribution function $\psi(e, \mathbf{f})$, one might ask to what approximation

$$
\psi(e, \mathbf{f}) \simeq \mathcal{F}(e, \mathbf{f})
$$

Accomplishing more than approximate strong equivalence is a priori unlikely, since this would amount to constructing an exact solution of the Boltzmann equation without actually solving (1.1). For practical applications however, weak equivalence suffices.

The Eddington factors $\mathbf{f}$ and $\overrightarrow{\mathbf{p}}$ are normalised moments of a non-negative weight function on the unit sphere and as such obey the Schwarz inequality $f^{2} \leq p \leq 1$, with $f \equiv|\mathbf{f}|$ and $p \equiv|\overrightarrow{\mathbf{p}} \cdot \mathbf{f} / f|$. In the diffusive (isotropic) limit $f=0, p=1 / 3$. In the ballistic (free-streaming) limit, the upper equality $f=p=1$ applies. With the aid of the energy conservation equation, the momentum conservation equation can be written as

$$
(-\nabla \ln e) / \kappa_{\text {tot }}=\mathbf{f} /\left(p-f^{2}\right) \equiv R
$$

where $\kappa_{\text {tot }}$ is the total inverse mean free path, including the artificial opacity (Cernohorsky and van Weert 1992; Dgani and Janka 1992). The effective Knudsen number $R$, the quotient of the mean free path and the energy scale height, is small $(R \ll 1)$ in the diffusive limit, and large $(R \gg 1)$ in the free-streaming limit. In the optically dense region the flux $\mathbf{F} \approx-\nabla e /\left(3 \kappa_{t o t}\right)$, in the optically thin regime $\mathbf{F} \approx e$.

Many closures or Eddington factors have been proposed: for reviews see e.g. (Janka 1991; Janka 1992; Janka, Dgani and van den Horn 1992; Levermore 1984; Minerbo 1978; Pomraning 1981), and references therein. Most closures compromise weak equivalence in favour of computational efficiency. Such pragmatism has been criticised by Janka, Dgani and van den Horn (1992), who found poor agreement between their exact Monte Carlo solutions of the neutrino transport equation and a number of one-dimensional closures. 
By one-dimensional closure we mean that $\vec{p}$ is a function of only one of the lower order Eddington factors, $e$ or $\mathbf{f}$.

Maximum entropy closure accounts for the radiation quantum statistics through its dependence on the occupation number density $e$ in addition to $f$. This makes the maximum entropy closure two-dimensional, including earlier one-dimensional closures as limiting cases. For Bose and for Fermi statistics, we find complementary two-dimensional Eddington surfaces, $p=p(e, f)$, which join in the classical limit.

We consider Bose, Fermi and classical radiation in a unified way. Formal symmetries emerge among these three types of radiation. In the next section, we present some general formalism and derive the common algorithm used to obtain the maximum entropy closures. In sections 3 and 4, we discuss the classical limit and the Bose-Einstein closures (MECBE). In Section 5, we consider the Fermi-Dirac closure (MEC-FD), with emphasis on limiting behaviour and global symmetries. These symmetries inspire the introduction of relative variables, in terms of which, MEC-FD exhibits universal scaling which leads to a simple analytic closure algorithm.

In the concluding sections, we argue that Monte Carlo transport calculations can be accounted for by MEC-FD and the range of occupation densities and fluxes expected during the early stages of neutron star formation. We dwell on the prospects of stronger equivalence and of future applications of the maximum entropy closure.

\section{Maximum Entropy Closure}

Throughout, we work within the spectral approach, in which all Eddington factors $e, f, p$ depend on the particle energy $\omega$. We restrict ourselves to systems with only one preferred spatial direction, relative to which vectors and tensors are defined. This includes spherically symmetric and rectangular geometries and enables us to consider only two independent quantities, the magnitude of the first Eddington factor $f= \pm|\mathbf{f}|$ and the forward component of the pressure tensor, $p \equiv|\overrightarrow{\mathbf{p}} \cdot \mathbf{f} / f|$.

The maximum entropy method constructs the least biased distribution based on limited available information: statistics, occupation density $e$ and flux $f$. The information content of a distribution is the negative of its entropy (Minerbo 1978). The information that the maximum entropy formalism introduces to the transport scheme mainly concerns the quantum statistics of the radiation. The maximum entropy distribution $\psi$ is found by 
maximising the entropy functional

$$
s[\psi] \propto(1-k \psi) \ln (1-k \psi)+k \psi \ln \psi
$$

with $k=1$ for Fermi-Dirac , $k=-1$ for Bose-Einstein and

$$
s[\psi] \propto \psi \ln \psi
$$

for Boltzmann statistics, under the constraints that the zeroth and first angular moment of the distribution function

$$
\begin{aligned}
& e=\frac{1}{4 \pi} \int_{0}^{2 \pi} d \phi \int_{-1}^{1} \psi d \mu \\
& f=\frac{1}{4 \pi e} \int_{0}^{2 \pi} d \phi \int_{-1}^{1} \mu \psi d \mu,
\end{aligned}
$$

equal the prescribed number density or phase space occupancy $e$ and the normalised flux $f$. The quantity $\mu=\cos \vartheta$ is the cosine of the normalised direction of the particle momentum with respect to the preferential spatial axis. The normalised forward pressure is analogously defined as

$$
p=\frac{1}{4 \pi e} \int_{0}^{2 \pi} d \phi \int_{-1}^{1} \mu^{2} \psi d \mu
$$

Following a standard procedure from the calculus of variations (Minerbo 1978), we introduce Lagrange multipliers $\eta, a$ for the constraints (2.3) and (2.4) respectively, and maximise

$$
S^{\prime}=\int d \mu s[\psi]+\eta \int d \mu \psi+a \int d \mu \mu \psi
$$

by considering its variation with respect to $\psi$. Absorbing signs and $\mu$-independent quantities into the Lagrange multipliers, we obtain the general form of the maximum entropy distribution function

$$
\psi(\mu)=\frac{1}{e^{\eta-a \mu}+k} .
$$

The particle statistics is indexed by the parameter $k=1,-1,0$. For Fermi-Dirac statistics $(k=1)$, the occupancy $0 \leq e \leq 1$. For Bose-Einstein $(k=-1)$ and Maxwell-Boltzmann $(k=0)$ statistics, $0 \leq e \leq \infty$. The requirement that the distribution be positive definite does not constrain the Lagrange multipliers $\eta$ and $a$ for Fermi and classical radiation, but imposes $0 \leq a<\eta \leq \infty$ for Bose statistics. Both quantum statistics reduce to the 
classical (Boltzmann) limit when the occupancy $e \ll 1$. The opposite limit is reached for $e \rightarrow \infty$ (high occupancy) in the Bose-Einstein case, and for $a \rightarrow \infty$ (maximum packing) in the Fermi-Dirac case. We restrict ourselves to outward directed radiation $f \geq 0$, so that $a \geq 0$. Because $f(\eta,-a)=-f(\eta, a)$, the treatment of backward-peaked radiation is straightforward.

The two constraints make the Lagrange multipliers $\eta$ and $a$ implicit functions of the number density $e$ and flux $f$. To arrive at a closure $p=p(e, f)$, we need to obtain $\eta(e, f)$ and $a(e, f)$ by inversion of the constraints $(2.3,2.4)$. To facilitate this inversion, previous authors approximated the maximum entropy distribution function and obtained various one-dimensional closures: Minerbo (1978) considered the maximum entropy distribution in the classical limit ( $e \ll 1$ )and found an analytic, but implicit, closure. In the Bose case, Pomraning (1981) and in the Fermi case, Cernohorsky, van den Horn and Cooperstein (1989) approximated (2.7), to obtain an explicit analytic inversion. Both these latter approximations lead to the one-dimensional Levermore-Pomraning Eddington factor.

We will now formulate the closure without approximating the angular distribution (2.7) in any way. The differential identity for $k \pm 1$,

$$
\psi \equiv \frac{k}{a} \frac{\partial g(\mu)}{\partial \mu}
$$

with $g(\mu) \equiv-\ln (1-k \psi)=\ln [1+k \exp (a \mu-\eta)]$, allows integrating by parts

$$
\int_{-1}^{1} \mu^{n} \psi d \mu=\frac{k}{a}\left[\left\{\mu^{n} g(\mu)\right\}_{-1}^{1}-n \int_{-1}^{1} \mu^{n-1} g(\mu) d \mu\right] \text {. }
$$

The first constraint with $n=0$ now follows without integration and can be inverted to express $\eta$ as a function of $e$ and $a$,

$$
\exp \eta \equiv B(e, a)=\frac{\sinh (1-k e) a}{\sinh (e a)},
$$

where $B$ is the reciprocal fugacity. This quantity has two useful symmetries:

$$
\begin{aligned}
B(e, a) & =B(e,-a), \\
B^{-1}(e, a) & =B(k-e, a) .
\end{aligned}
$$

The first symmetry insures backward-forward symmetry $f(e,-a)=-f(e, a)$. In the BoseEinstein case, the second reciprocal symmetry relates physical states $e>0$ to unphysical ones $-(1+e)<0$, and has no physical consequences. However, in the Fermi-Dirac case, $e$ 
and $1-e$ are both positive. In section 5 , we will see that these symmetries point the way to an analytic form for the variable Eddington factor $p=p(f, e)$.

With $\eta$ as a function of $e$ and $a$, one Lagrange multiplier is eliminated from the remaining two Eddington factors $f$ and $p$. Janka, Dgani and van den Horn (1992) stop at this level to discuss and plot the Eddington factors $f, p$ as functions of $e, a$. However, we use Eq. (2.9) with $n=1$ to eliminate the second Lagrange multiplier $a$ by numerically inverting the second constraint (2.4). The remaining integral is numerically more tractable than the original one in equation (2.4), and is solved with a Gauss-Legendre quadrature. For given $e$ and $f$, the inversion of constraint (2.4) is now a one-dimensional root-finding problem, which we solve by a combination of a False Position and Newton-Raphson algorithm.

Having obtained the root $a(e, f)$ of (2.4), equation (2.9) with $n=2$ yields $p(e, f)$. With this procedure, we have an algorithm that inputs $e, f$, and returns the two-dimensional variable Eddington factor $p(e, f)$.

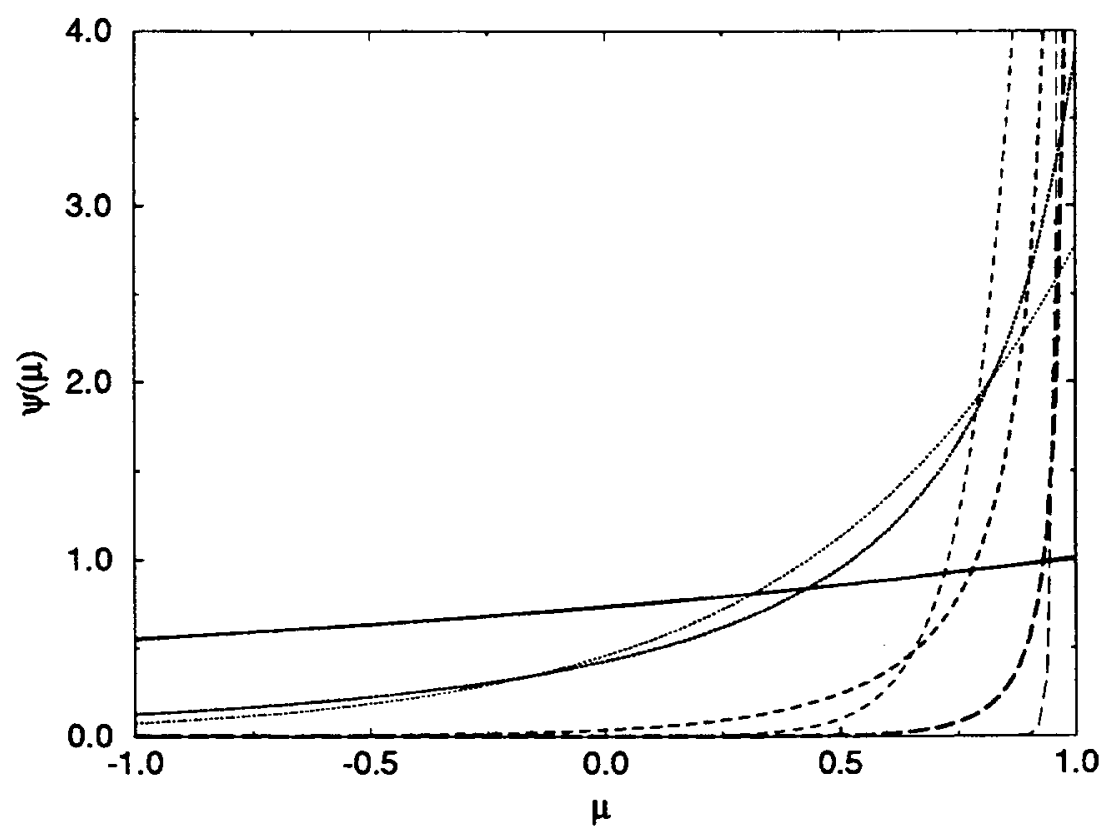

Figure 1: Maximum entropy Bose-Einstein (heavy curves) and Maxwell-Boltzmann (light curves) distributions for $e=0.75$ and four values of the normalised flux $f=0.1(-), 0.5$ $(\cdots), 0.9(--)$ and $0.99(---)$. The Bose distribution is always more forward-peaked than the classical one. 


\section{Maximum Entropy Closure in the Classical Limit}

With either Bose or Fermi statistics, when the occupation $e \ll 1, B \approx \sinh a / e a$ becomes very large, and the quantum index $k$ in the denominator of $\psi$ can be neglected. With $k=0$, the integrals $(2.4,2.5)$ can be performed analytically, and lead to Minerbo's flux-limiter (Minerbo 1978)

$$
f(a)=L(a), \quad p(a)=1-2 f(a) / a,
$$

where $L(q) \equiv \operatorname{coth} q-1 / q$ is the Langevin function and $q(\mathrm{~L})$ is its inverse. The Eddington factors $p, f$ each depend on only one Lagrange multiplier, and the implicit variable Eddington factor $p=p(f)=1-2 f / q(f)$ is a single limiting curve separating MEC-BE and MEC-FD.

\section{Maximum Entropy Closure for Bose-Einstein Radiation}

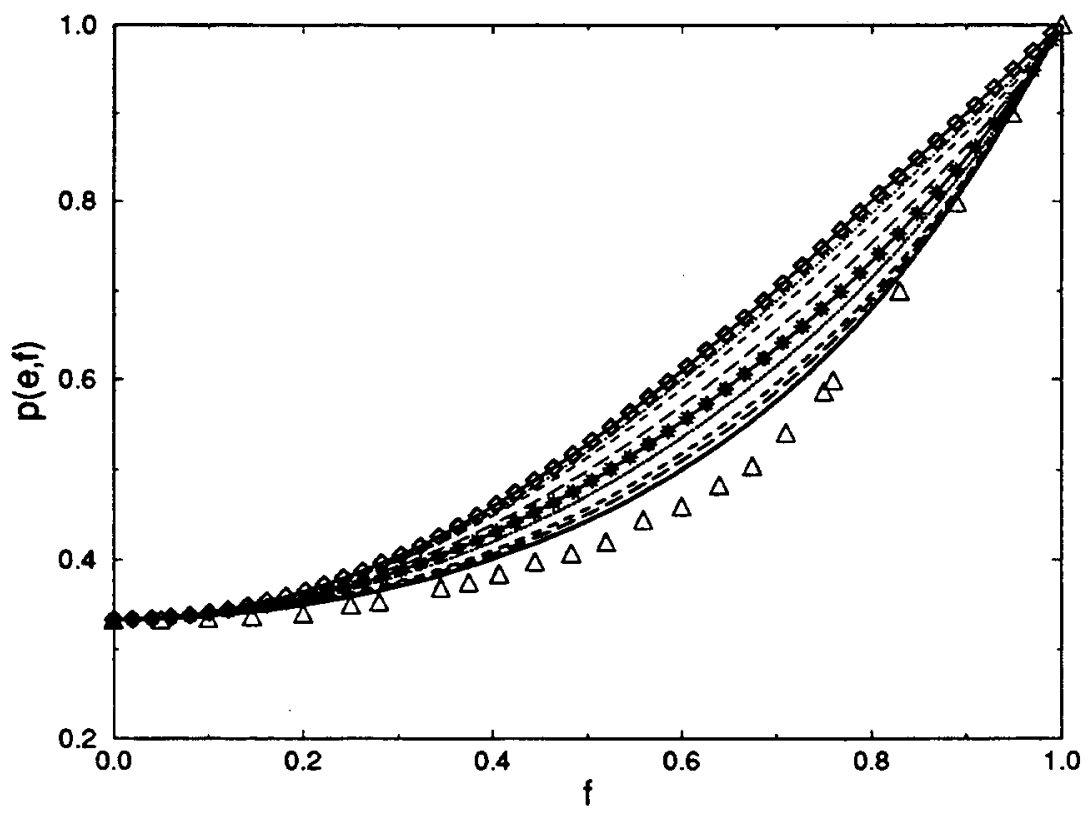

Figure 2: Maximum entropy Bose-Einstein closure for nine occupancies bottom-to-top $e=0,0.1,0.2,0.5,1 .(*), 2 ., 5 ., 10, \infty(\diamond)$. Bose-Einstein closure allows a family of trajectories between the Maxwell-Boltzmann (bottom-) and Levermore-Pomraning (top $\diamond)$ trajectories. The exact energy-averaged neutrino trajectory obtained by Janka $(\Delta)$ lies below the range of MEC-BE 
The distribution function (2.7) must be positive definite, which constrains the Lagrange multipliers to be $0 \leq a<\eta \leq \infty$, and their ratio $z \equiv \eta / a>1$. For small occupation density $e \ll 1$, the distribution tends to the Boltzmann limit discussed in the preceding Section.

For large occupation density $(e \gg 1)$ the denominator of $\psi(\mu)$ is small. Hence the exponent $a(z-\mu) \ll 1$ : both Lagrange multipliers must be small individually $a<\eta \ll 1$, but their ratio $1<z \ll 1 / a \rightarrow \infty$ can still be large.

In this regime, we may expand the exponential in a Taylor series and approximate the distribution function

$$
\psi(\mu) \approx \frac{1}{a(z-\mu)}, e \gg 1
$$

In the constraints $(2.3,2.4)$ and in equation $(2.5)$, the angular integrals may be performed analytically and lead to

$$
p=z f(z) \quad, \quad f(z)=z-2\left[\log \left(\frac{z+1}{z-1}\right)\right]^{-1} .
$$

Setting $z=\operatorname{coth} R$,

$$
p=f \operatorname{coth} R=f\left(f+R^{-1}\right) \quad, \quad f=L(R)=\operatorname{coth} R-1 / R
$$

This limiting trajectory, the 'logarithmic limit' discussed by Fu (1987), is none other than the Levermore-Pomraning closure (Levermore and Pomraning 1981). Its streaming behaviour, containing both the isotropic diffusion and the ballistic limits, is controlled by the ratio $z \equiv \eta / a$ of the Lagrange multipliers for a given value $a \ll 1$.

When $z \gg 1=\max (|\mu|),(1 \gg \eta \gg a)$, the distribution (4.1) approaches the isotropic limit, $R \approx(1 / z), f \ll 1, p \approx 1 / 3$. When $z_{+} \rightarrow 1,\left(\eta_{+} \rightarrow a\right)$ the opposite ballistic limit is reached, in which the distribution (4.1) peaks in the forward direction $\mu=1$ as $z \approx 1$ and $R \rightarrow \infty, f \approx p \approx 1$.

For intermediate values of $e$ and $f$, we find the maximum entropy distributions $\psi(e, f)$, and the variable Eddington factor $p=p(f, e)$ by the numerical inversion described in Section 2. Figure 1 shows, for a fixed $e=0.75$, the Bose-Einstein maximum entropy distribution $\psi(\mu)$, for a range of fluxes $f$. Comparison with the Maxwell-Boltzmann distributions shows that the Bose statistics considerably enhances the forward peaking. Figure 2 shows the MEC-BE surface, parametrised by the occupancy $e$, and bounded by MEC-MB for $e \ll 1$ and the Levermore-Pomraning trajectory for $e \gg 1$. 


\section{Maximum Entropy Closure for Fermi-Dirac Radiation}

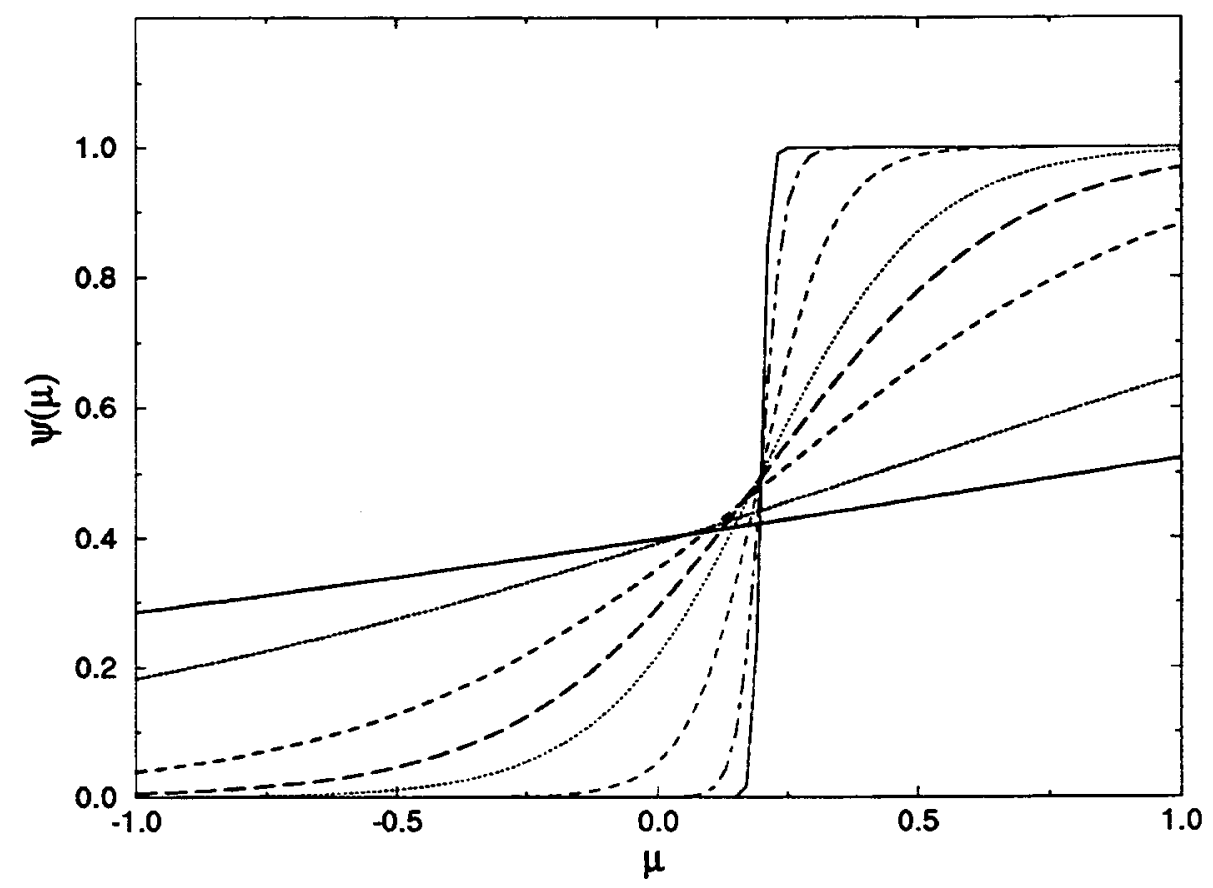

Figure 3: Maximum entropy FD distribution $\psi(\mu)$ for occupancy $e=0.4$. and the allowed fluxes $=0.1(--), 0.2,0.4,0.5,0.55,0.59,0.599,0.5999(-)$. The sharpness of the step at $\mu=1-2 e$ increases with the flux.

Equation (2.10) implies that in the limit $a \rightarrow \infty, \eta \simeq(1-2 e) a$, the distribution function approaches an angular step-function with Fermi surface at $\mu=(1-2 e)$ and reciprocal 'angular temperature' $a$. Because of this step-function behaviour, we call this as the extreme degeneracy limit. In this case the integrals $(2.4,2.5)$ can be done analytically, and give the maximal forward angular packing limits for the flux and pressure, allowed by the exclusion principle, $f_{\max }=1-e$ and $p_{\max }=1-2 e+(4 / 3) e^{2}$ (Janka, Dgani and van den Horn 1992).

For arbitrary degeneracy, we go over to relative coordinates $x$ and $\chi$ defined as

$$
x(e, a) \equiv \frac{f(e, a)}{f_{\max }(e)} \quad, \quad \chi(e, a) \equiv \frac{p(e, a)-1 / 3}{p_{\max }(e)-1 / 3},
$$

and referred to as 'flux-saturation' and 'pressure-saturation' respectively. The relative coordinates run from zero to one as a goes from zero to infinity, and the radiation field from isotropic to forward packed. Due to the symmetries $(2.11,2.12) x$ and $\chi$ are invariant 
under the transformation $e \rightarrow 1-e$, at fixed $a$ :

$$
x(e, a)=x(1-e, a) \quad, \quad \chi(e, a)=\chi(1-e, a) .
$$

Figure 4 shows the maximum entropy Eddington surface $p(f, e)$ labeled by $e$ and bounded

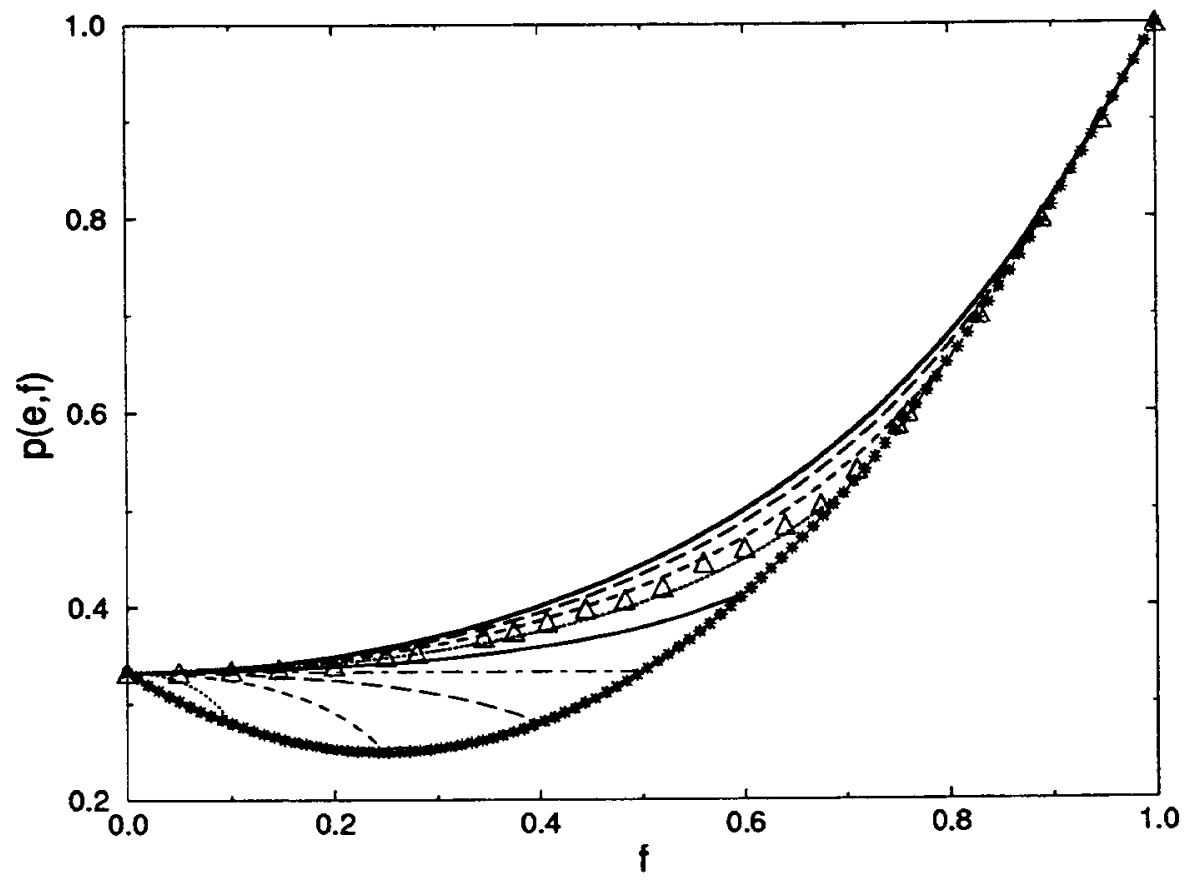

Figure 4: MEC-FD surface $p(e, f)$. The uppermost curve $(-)$ is the classical limit $e \rightarrow 0$. The bottom curve $((* \cdots *)$ is the maximal packing envelope. Between these lie from top to bottom $e=0.1,0.2,0.3,0.4,0.5(--), 0.6,0.75,0.9$. The energy-averaged Eddington factors from Janka's Monte Carlo calculations are marked by $\triangle$

by the Boltzmann and maximum-packing curves. The maximal-packing curve consists of the points $\left(f_{\max }(e), p_{\max }(e)\right)$ for a range of values of $0<e<1$.

The high packing and diffusive limits of the closure are easily derived. Near the degenerate limit, $a \gg 1$, the distribution function may be approximated by the Sommerfeld expansion (Balian 1991) $\psi(a, z, \mu) \approx \theta\left((\mu-z)-\left(\pi^{2} / 6 a^{2}\right) \delta^{\prime}(\mu-z)+\cdots \quad\right.$, with $a z=\eta$ and $\delta^{\prime}(x)$ the derivative of the Dirac $\delta$-function with respect to its argument. As functions of $a$, the Eddington factors approach saturation as

$$
x(e, a) \approx 1-A a^{-2}, \chi(e, a) \approx 1-3 A a^{-2},
$$


with $A(e)=\pi^{2} /(12 e(1-e))$, so that after elimination of $a$

$$
\chi(x)=3 x-2 \quad, x \rightarrow 1
$$

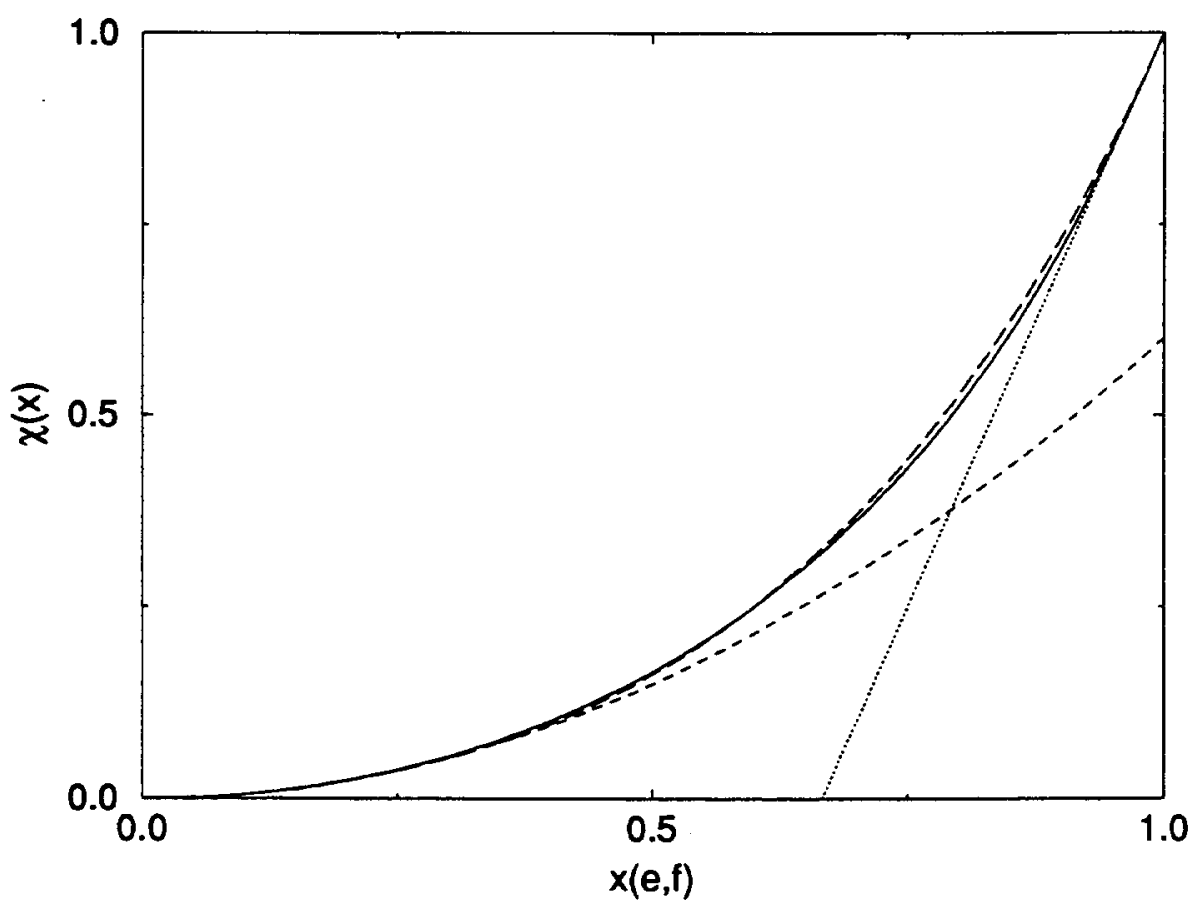

Figure 5: The universal variable pressure-saturation $\chi(x)$, equation (5.7) as a function of the flux-saturation $x$. The dotted line is the free-streaming limit $\chi(x \rightarrow 1) \approx 3 x-2$, the dashed line is the diffusive limit $\chi(x \rightarrow 0) \approx 3 x^{2} / 5$. The long-dashed curve is the polynomial fit, equation (5.9).

In the nearly isotropic limit, $a \ll 1$, the exponential in the denominator of the distribution function can be expanded in a Taylor series (Cernohorsky, van den Horn and Cooperstein 1989; Cernohorsky and van Weert 1992). This leads to the Levermore-Pomraning closure (4.3), with now $R \equiv e a B=f /\left(p-f^{2}\right)$. Because this expansion is only justified in the isotropic limit, $a \ll 1$, the Levermore-Pomraning closure for Fermi-Dirac radiation is only a reasonable approximation for small flux(-saturation), as can be clearly ascertained from figure 4 . In terms of the relative variables, the Eddington factors approach their diffusive values as

$$
x(e, a) \approx a / 3 \quad, \chi(e, a) \approx a^{2} / 15
$$


so that

$$
\chi(x)=3 x^{2} / 5 \quad, \quad x \ll 1 \quad .
$$

After $a$ is eliminated, how is the $e \rightarrow 1-e$ invariance expressed in the variable Eddington factor $p(e, f)$ ? After the Lagrange multiplier $a$ is eliminated numerically in equation (5.1) to express the variable pressure-saturation

$$
\chi(e, x) \equiv \frac{p\left(e, x f_{\max }(e)\right)-1 / 3}{p_{\max }(e)-1 / 3}
$$

as function of the flux saturation $x, \chi(e, x)$ turns out to be not only $e \rightarrow 1$-e-invariant, but entirely independent of $e$, a universal function of $x$ only, (solid curve in figure 5). Hence, its analytical expression must also hold for for small $e$, where $f_{\max } \approx 1, p_{\max } \approx 1$, and the the variable Eddington factor $p(e, f)=p(x)$ is given by its classical limit (3.1). Substituting this in (5.7) gives

$$
\chi(x)=1-3 x / q(x),
$$

which matches the numerical curve in figure 5. Here, $q(x)$ is the inverse of the Langevin function $x=\operatorname{coth} q-1 / q$. The lowest order polynomial approximation to this universal curve which has the correct behaviour $(5.4,5.6)$ in both limits and contains no free parameters, is

$$
\chi(x)=x^{2}\left(3-x+3 x^{2}\right) / 5,
$$

to $2 \%$ accuracy (long-dashed curve in figure 5 ).

From equation (5.7), the variable Eddington factor

$$
p(e, f)=\frac{2(1-e)(1-2 e)}{3} \chi\left(\frac{f}{1-e}\right)+\frac{1}{3},
$$

is thus expressed in terms of the universal pressure saturation. Either the exact expression (5.8) or its approximation, equation (5.9) perfectly reproduces the variable Eddington factor surface shown in Fig. 4. The relative error of the polynomial approximation is at worst of the order of a few per thousand, sufficient for most if not all applications. The laborious numerical inversion needed for the Bose-Einstein Eddington factors can, in the Fermi-Dirac case, be circumvented by a ten-line algorithm.

\section{Comparison with Monte Carlo Eddington Factors}

Superimposed on the maximum entropy Eddington surface in figures 2 and 4 are energyaveraged variable Eddington factors calculated from exact Monte Carlo solutions of the 
neutrino transport equation, for a variety of material backgrounds, during the early neutrino-cooling phase of neutron star formation (Dgani and Janka 1992; Janka 1991; Janka 1992; Janka, Dgani and van den Horn 1992). These energy-averaged exact Eddington factors fall in a narrow bound about $e=0.25-0.3$ for $f<0.7$ and near the maximal packing envelope for $f>0.7$, within the MEC-FD region in Fig. 4 and entirely outside the MEC-BE region in Fig. 2.

At least two factors contribute to this apparently remarkable universal behaviour of the Monte Carlo Eddington factors. First, they are energy averages, so that the Monte Carlo spectral Eddington factors would be scattered in the $p-f$-plane, but, if weak equivalence holds, would still lie on the MEC-FD surface. For this evolutionary epoch and stellar environment, the main contribution to the energy average apparently comes from bins with moderate occupation density, $e(\omega) \sim 0.2-0.3$ and moderate flux. At higher fluxes $f>0.7$, bins with even smaller occupation density $e<0.1$ dominate. This explains why Minerbo's Boltzmann closure performs rather well over a range of Monte Carlo Eddington trajectories.

Second, the Monte Carlo calculations were performed in semi-transparent regions of stellar models, several seconds after bounce, during the early phases of the hydrostatic neutron star formation, when the delayed explosion mechanism presumably operates. The shock wave has by then traversed the semi-transparent region, dissociated the heavy nuclei present, and left a mixture of nucleons and $\alpha$ 's. The chemical compositions and the matter density profiles determining the neutrino opacity, must therefore be similar in all models. This largely explains the model-to-model universality.

Under atypical conditions, when narrow shell sources in the semi-transparent region occur together with a steep gradient in opacity, so that large occupation densities coincide with high fluxes, Janka reports $p<1 / 3$. In such special circumstances, the Monte Carlo results could be qualitatively well approximated by MEC-FD for $e>1 / 2, f \rightarrow f_{\max }$, but could not be described by any one-dimensional closure or direct phenomenological fit to the Monte Carlo data such as (Dgani and Janka 1992; Janka 1991).

Comparison between figure 3 and figures in (Janka 1991; Janka 1992; Janka, Dgani and van den Horn 1992) of local Monte Carlo energy-integrated neutrino distributions $\int \omega^{3} \mathcal{F}(\omega, \mu) d \omega$ as function of $\mu$, indicates that our MEC-FD distribution approximates the exact distribution, at least qualitatively . Of course, the exact spectral distribution must tend to an angular step-function with increasing degeneracy, just as the MEC-FD 
distribution. Because Janka, Dgani and van den Horn (1992) present energy-averaged Eddington factors and do not indicate the e-dependence of their Eddington factors, we cannot at this point further investigate weak and strong equivalence to spectral MEC-FD. It would however, seem worthwhile to study weak and strong equivalence between Monte Carlo and MEC-FD calculations on the same stellar background.

\section{Conclusion}

We have implemented the unapproximated maximum entropy closure into the analytic expression (5.10) for Fermi radiation and a numerical inversion algorithm for Bose radiation.

For neutrino radiation, MEC-FD reproduces and helps explain the exact Monte Carlo radiation transport during neutron star formation. Because the variable Eddington factor depends on occupancy $e$ as well as flux $f$, it can better approximate the Monte Carlo neutrino radiation transport in various environments than any one-dimensional fit or closure does. The Levermore-Pomraning variable Eddington factor, being the high occupation (e $\gg 1)$ Bose limit, is the worst possible choice for a neutrino transport closure in the semi-transparent and free streaming regimes (Janka, Dgani and van den Horn 1992). Only in the near-isotropic diffusive limit it is a reasonable approximation to the MEC-FD closure or to Monte Carlo results.

Bose-Einstein statistics enhances forward peaking, allows for condensation into a single angular state and thus enhances the effects of short-range interactions. In astrophysical environments, such as stellar atmospheres, where the radiation field is far from local thermodynamic equilibrium, the photon occupation density is generally low $(e \ll 1)$ so that the classical limit (Minerbo 1978) will usually be the best and most efficient statistical closure. The Levermore-Pomraning Eddington factor, the high occupation limit of MEC$\mathrm{BE}$, should generally give less realistic results. It remains to be seen whether MEC-BE will be as useful as MEC-FD promises to be. 
Acknowledgement This work was supported by the Director, Office of Energy Research, Office of High Energy and Nuclear Physics, Division of Nuclear Physics, of the U.S. Department of Energy under Contract DE-AC03-76SF00098 at the Lawrence Berkeley Laboratory and by the Department of Energy contract AC02-76-ERO-3071 at the University of Pennsylvania. S.A.B. thanks the Center for Particle Astrophysics and the Nuclear Science Theory Division of the Lawrence Berkeley Laboratory for hospitality during the summer of 1993. J.C. thanks the Max-Planck Institut für Astrophysik for the use of their computing resources.

\section{References}

Balian, R. 1991, From Microphysics to Macrophysics, vol. 2, Springer Verlag

Cernohorsky, J., van den Horn, L. J. and Cooperstein, J. 1989, J. Quant. Spectros. Rad. Transf. , 42, 603

Cernohorsky, J. and van Weert, C. G. 1992, ApJ , 398, 190

Dgani, R. and Janka, H. -T. 1992, Astr. Ap. , 256, 428

Fu, A. 1987, ApJ , 323, 211

Janka, H. -T. 1991, Neutrino Transport in Type II Supernovae and Protoneutron Stars by Monte Carlo Methods, Max-Planck Institut für Astrophysik, F.R.G., PhD Thesis 1992, Astr. Ap. , 256, 452

Janka, H. -T., Dgani, R. and van den Horn, L. J. 1992, Astr. Ap. , 265, 345

Levermore, C. D. 1984, J. Quant. Spectros. Rad. Transf. , 31, 149

Levermore, C. D. and Pomraning, G. C. 1981, ApJ , 248, 321

Minerbo, G. N. 1978, J. Quant. Spectros. Rad. Transf. , 20, 541

Pomraning, G. C. 1981, J. Quant. Spectros. Rad. Transf., 26, 385 